\title{
Complex Dynamical Behavior of a Two-Stage Colpitts Oscillator with Magnetically Coupled Inductors
}

\author{
V. Kamdoum Tamba, ${ }^{1,2}$ H. B. Fotsin, ${ }^{1}$ J. Kengne, ${ }^{3}$ F. Kapche Tagne, ${ }^{2}$ and P. K. Talla \\ ${ }^{1}$ Department of Physics, Laboratory of Electronics and Signal Processing (LETS), Faculty of Science, University of Dschang, \\ P.O. Box 67, Dschang, Cameroon \\ ${ }^{2}$ Department of Telecommunication and Network Engineering, IUT-Fotso Victor of Bandjoun, University of Dschang, \\ P.O. Box 134, Bandjoun, Cameroon \\ ${ }^{3}$ Department of Electrical Engineering, Laboratory of Automation and Applied Computer (LAIA), \\ IUT-Fotso Victor of Bandjoun, University of Dschang, P.O. Box 134, Bandjoun, Cameroon \\ ${ }^{4}$ Department of Physics, Laboratory of Mechanics and Modelling of Physical Systems (L2MPS), Faculty of Science, \\ University of Dschang, P.O. Box 67, Dschang, Cameroon
}

Correspondence should be addressed to H. B. Fotsin; hbfotsin@yahoo.fr

Received 26 May 2014; Accepted 11 September 2014; Published 21 October 2014

Academic Editor: Uchechukwu E. Vincent

Copyright @ 2014 V. Kamdoum Tamba et al. This is an open access article distributed under the Creative Commons Attribution License, which permits unrestricted use, distribution, and reproduction in any medium, provided the original work is properly cited.

\begin{abstract}
A five-dimensional (5D) controlled two-stage Colpitts oscillator is introduced and analyzed. This new electronic oscillator is constructed by considering the well-known two-stage Colpitts oscillator with two further elements (coupled inductors and variable resistor). In contrast to current approaches based on piecewise linear (PWL) model, we propose a smooth mathematical model (with exponential nonlinearity) to investigate the dynamics of the oscillator. Several issues, such as the basic dynamical behaviour, bifurcation diagrams, Lyapunov exponents, and frequency spectra of the oscillator, are investigated theoretically and numerically by varying a single control resistor. It is found that the oscillator moves from the state of fixed point motion to chaos via the usual paths of period-doubling and interior crisis routes as the single control resistor is monitored. Furthermore, an experimental study of controlled Colpitts oscillator is carried out. An appropriate electronic circuit is proposed for the investigations of the complex dynamics behaviour of the system. A very good qualitative agreement is obtained between the theoretical/numerical and experimental results.
\end{abstract}

\section{Introduction}

During the last three decades, a tremendous attention has been devoted to design chaotic electronic oscillators. The focus on this interesting research field comes mainly from two facts: first, one can observe chaos and can also control the dynamics of the oscillator by simply changing the physically accessible parameters of the oscillator, for example, linear resistor, linear capacitor, voltage levels, coupled inductors, and so forth; second, there are a multitude of applications of chaotic electronic oscillators starting from chaotic electronic secure communication to cryptography [1]. In this regard, the classical Colpitts oscillator with single transistor was investigated at $1 \mathrm{KHz}$ frequency [2], high $(3-300 \mathrm{MHz})$ frequencies [3], and ultrahigh $(300-1000 \mathrm{MHz})$ frequencies [4] using both numerical and experimental methods. The interest devoted to this oscillator is motivated by its simple physical realization and low power requirement. Nevertheless, the main limitation of the classical Colpitts oscillator is its incapacity to exhibit higher fundamental frequencies in chaotic regime [5]. In order to solve this problem, alternatives to this standard version of the Colpitts oscillator, namely, the two-stage and improved version, were reported in [6, 7]. In comparison to a single stage Colpitts oscillator, the two-stage Colpitts oscillator presents better spectral properties which are suitable for communication application. Some interesting works $[8,9]$ have been reported concerning the dynamics and the control of chaos in two-stage Colpitts oscillator using the 
ideal current source (i.e., power supply) as a control parameter which is not easily accessible in practical situations. Thus, it is difficult to control the dynamics of the two-stage Colpitts oscillator using one direct accessible electrical component. To overcome this problem, the present work proposes a controlled version of a two-stage Colpitts oscillator using a pair of coupled inductors and a linear resistor which is easy to use as a control parameter. The control scheme reported in [10] exploits the coupling between the passive network and the chaotic Colpitts oscillator through mutual inductors. This technique requires a simpler control circuit, which has the advantage of direct and easy implementation. The single resistor is used to control chaos in the Colpitts oscillator. This control technique is exploited in this paper to investigate the rich dynamics of two-stage Colpitts oscillator. However, it could also be used as a mean for parameter modulation strategy for secure communication applications. On the other hand, previous works [11-14] related to the investigation of the dynamics of this type of oscillators are based on piecewise linear models (PWL) though restricting to the first-order description of the system's dynamics [8, 15]. In fact, the PWL model represents only a first-order approximation of the reality; therefore it may give rise to different types of bifurcations compared to those exhibited by the real oscillator. In contrast to previous mathematical analysis, the present work proposes a smooth (exponential model) mathematical model to investigate the nonlinear dynamics and chaos in the controlled two-stage Colpitts oscillator. Various bifurcation diagrams and corresponding graphs of Lyapunov exponents are provided to characterize the dynamics of the system in terms of the control parameter (single variable resistor). Finally, the effects of transistor gain on the dynamics of the controlled two-stage Colpitts oscillator are analyzed.

The layout of this paper is as follows. Section 2 is devoted to the circuit description and mathematical model of the controlled two-stage Colpitts oscillator. The circuit diagram of the oscillator is addressed and the corresponding mathematical model is derived. Section 3 deals with the dynamical properties of controlled two-stage Colpitts oscillator. Dissipation and existence of attractors, bifurcation analysis, chaotic behavior, and interior crisis are investigated. In Section 4, experimental investigations of the dynamical behaviour of the system are described. Finally, some concluding remarks are given in Section 5 .

\section{Circuit Description and Mathematical Model}

2.1. Circuit Description. The simplest circuit diagram of the controlled two-stage Colpitts oscillator is depicted in Figure 1(a). The circuit contains two bipolar junction transistors (BJT) $Q_{1}$ and $Q_{2}$ used in the common base configuration as the nonlinear gain element. The resonant network consists of coupled inductors $L_{1}$ and $L_{2}$, mounted in series with the biasing and damping resistor $R$, and three capacitors $\left(C_{1}, C_{2}\right.$, and $\left.C_{3}\right) . R_{L}$ is a variable resistor which is used as a control parameter of the oscillator. The ideal current generator $I_{0}$ is used to maintain constant biasing emitter current. It is important to note that, in the circuit diagram, the only nonlinear devices are the bipolar junction transistors $\left(Q_{1}\right.$ and $\left.Q_{2}\right)$, which are responsible for the striking complex behavior exhibited by the oscillator. The BJT model consists of a nonlinear voltage-controlled resistance $R_{E}$ and a linear current-controlled current source $I_{E}$ as shown in Figure 1(b). The main difference between the classical circuit diagram of the two-stage Colpitts oscillator and the controlled version is the presence of coupled inductors and variable resistor in the collector node of the transistor $Q_{1}$.

2.2. Mathematical Model. To model the circuit, some assumptions are considered. Firstly, we assume that the capacitors, the inductor, and the resistor of the resonant network are linear. Secondly, we neglect parasitic capacitors $C_{b e}$ and $C_{b c}$. Thirdly, the transistors $\left(Q_{1}\right.$ and $\left.Q_{2}\right)$ are modeled as in [15]. The V-I characteristic of the nonlinear resistor $R_{E}$ is defined as usual by

$$
I_{E}=f\left(V_{\mathrm{BE}}\right)=I_{S}\left(\exp \left(\frac{V_{\mathrm{BE}}}{V_{T}}\right)-1\right),
$$

where $I_{E}$ is the emitter current, $V_{\mathrm{BE}}$ is the voltage across the B-E junction, $I_{S}$ is the saturation current of the B-E junction, $V_{T}=K_{b} T / e$ is a thermal voltage with $K_{b}$ the Boltzmann constant, $T$ is the absolute temperature, and $e$ is the elementary charge. At room temperature, $V_{T}$ is approximately equal to $26 \mathrm{mV}$. Taking into account the BJT model of Figure 1(b) and denoting $I_{1}$ and $I_{2}$ as the current flowing through the inductors $L_{1}$ and $L_{2}$, respectively, and $V_{C_{i}}(i=1,2,3)$ as the voltage across capacitor $c_{i}(i=1,2,3)$, the state equations of the circuit of Figure 1(a) exploiting the Kirchhoff electric circuit law are the following:

$$
\begin{aligned}
c_{1} \frac{d V c_{1}}{d t}= & I_{1}-\alpha_{F_{1}} f\left(V_{\mathrm{BE} 1}\right) \\
c_{2} \frac{d V c_{2}}{d t}= & I_{1}-I_{0}+\left(1-\alpha_{F_{1}}\right) f\left(V_{\mathrm{BE} 1}\right) \\
& +\left(1-\alpha_{F_{2}}\right) f\left(V_{\mathrm{BE} 2}\right) \\
c_{3} \frac{d V c_{3}}{d t}= & I_{L}+\left(1-\alpha_{F_{1}}\right) f\left(V_{\mathrm{BE} 1}\right)-\alpha_{F_{2}} f\left(V_{\mathrm{BE} 2}\right)
\end{aligned}
$$

$$
\begin{aligned}
& \frac{L_{1} L_{2}-M^{2}}{L_{2}} \frac{d I_{1}}{d t}=V_{0}-V_{C_{1}}-V_{C_{2}}-V_{C_{3}}-R I_{1}-\frac{M}{L_{2}} R_{L} I_{2} \\
& \frac{L_{1} L_{2}-M^{2}}{M} \frac{d I_{2}}{d t}=V_{0}-V_{C_{1}}-V_{C_{2}}-V_{C_{3}}-R I_{1}-\frac{L}{M} R_{L} I_{2}
\end{aligned}
$$

where $\alpha_{F_{i}}(i=1,2)$ is common base forward short-circuit current gain of the transistors, $M=k \sqrt{L_{1} L_{2}}$ represents the mutual inductance between $L_{1}$ and $L_{2}$, given the coupling factor $k$, and $V_{\mathrm{BE}_{1}}=V_{1}-V_{C_{2}}-V_{\mathrm{C}_{3}}$ and $V_{\mathrm{BE}_{2}}=-V_{\mathrm{C}_{2}}$ are, respectively, the base-emitter voltage of transistors $Q_{1}$ and 


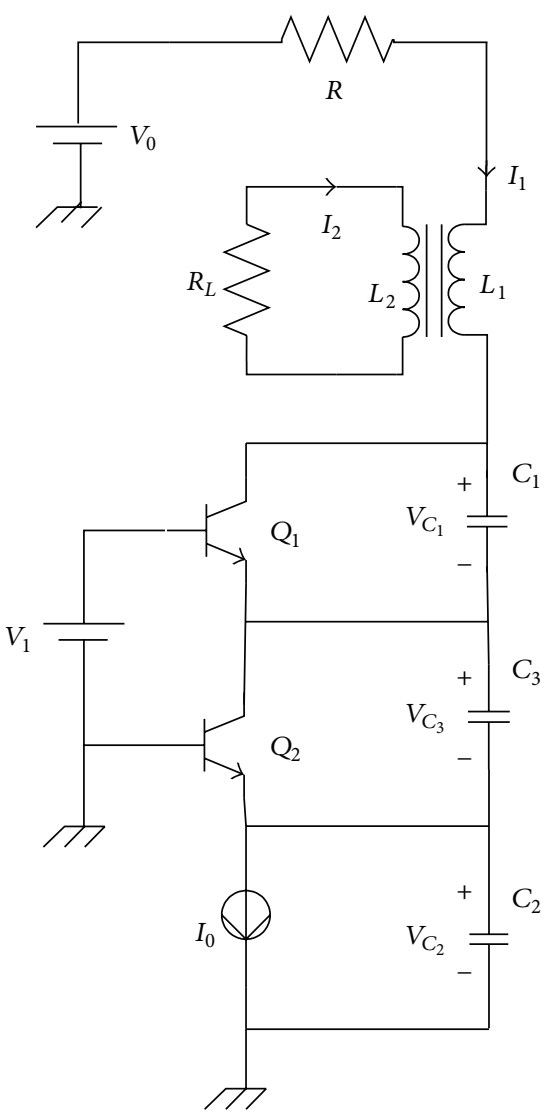

(a)

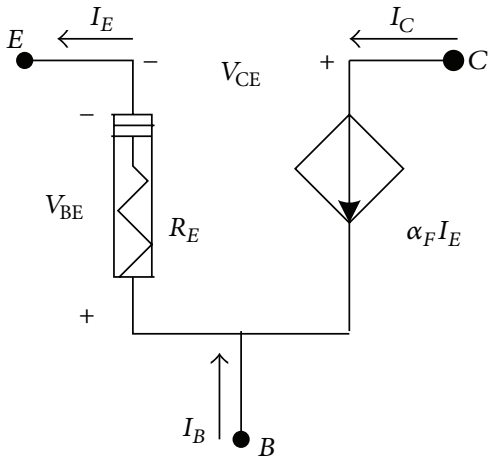

(b)

Figure 1: (a) Physical realization of the controlled two-stage Colpitts oscillator: the circuit parameters are $C_{1}=8 \mathrm{nF} ; C_{2}=C_{3}=10 \mathrm{nF}$; $L_{1}=16 \mathrm{uH} ; L_{2}=23 \mathrm{uH} ; R=35 \Omega ; V_{0}=12 \mathrm{~V} ; V_{1}=6 \mathrm{~V} ; I_{0}=1.750 \mathrm{~mA}$; and $R=60 \Omega$ potentiometer. The BJT model (b) consisting of a current-controlled current source and a single diode.

$Q_{2}$. For simplicity, we first assume that the common base forward short-circuit gain of the transistor $\alpha_{F_{i}}=1(i=1,2)$; that is, we neglect the base current. Equation (2a)-(2e) has a single equilibrium point $\left(V_{C_{1}}^{0}, V_{\mathrm{C}_{2}}^{0}, V_{\mathrm{C}_{3}}^{0}, I_{1}^{0}, I_{2}^{0}\right)^{T}$ which can be obtained by setting the right-hand side of $(2 \mathrm{a}),(2 \mathrm{~b}),(2 \mathrm{c})$, (2d), and (2e) to zero. The following expressions are obtained:

$$
\begin{aligned}
V_{C_{1}}^{0} & =V_{0}-V_{1}-R I_{0}+V_{T} \ln \left(1+\frac{I_{0}}{I_{s}}\right), \\
V_{C_{2}}^{0} & =-V_{T} \ln \left(1+\frac{I_{0}}{I_{s}}\right), \\
V_{C_{3}}^{0} & =V_{1}, \\
I_{1}^{0} & =I_{0}, \\
I_{2}^{0} & =0 .
\end{aligned}
$$

For convenient numerical analyses, let us introduce the following set of dimensionless state variables and parameters:

$$
\begin{aligned}
& x_{i} V_{T}=V_{C_{i}}-V_{C_{i}}^{0} \quad(i=1,2,3), \\
& x_{j} V_{T}=\rho\left(I_{j}-I_{j}^{0}\right) \quad(j=4,5),
\end{aligned}
$$

$$
\begin{aligned}
t=\tau \sqrt{L C_{2}}, & \rho & =\sqrt{\frac{L_{1}}{C_{2}}}, \\
\sigma_{1}=\frac{C_{2}}{C_{1}}, & \sigma_{2} & =\frac{C_{2}}{C_{3}}, \\
\sigma_{3}=\frac{L_{1} L_{2}}{\left(L_{1} L_{2}-M^{2}\right)}, & \sigma_{4} & =\frac{L_{1} M}{\left(L_{1} L_{2}-M^{2}\right)}, \\
\gamma=\frac{\rho I_{0}}{V_{T}}, & \varepsilon & =\frac{R}{\rho}, \\
\mu=\frac{L_{1} L_{2}}{M^{2}}, & \alpha & =\frac{R_{L} M}{L_{2} \rho} .
\end{aligned}
$$

The physical parameters of controlled two-stage Colpitts oscillator are $C_{1}=8 \mathrm{nF} ; C_{2}=C_{3}=10 \mathrm{nF} ; L_{1}=16 \mathrm{uH}$; $L_{2}=23 \mathrm{uH} ; R=35 \Omega ; V_{0}=12 \mathrm{~V} ; V_{1}=6 \mathrm{~V} ; I_{0}=1.750 \mathrm{~mA}$; and $R_{L}=60 \Omega$ potentiometer.

Therefore, the above equations (2a)-(2e), according to (4), are rewritten in the dimensionless form as

$$
\dot{x}_{1}=\sigma_{1}\left(x_{4}-\gamma \phi\left(x_{2}+x_{3}\right)\right) \text {, }
$$




$$
\begin{aligned}
& \dot{x}_{2}=x_{4}, \\
& \dot{x}_{3}=\sigma_{2}\left(x_{4}-\gamma \phi\left(x_{2}\right)\right), \\
& \dot{x}_{4}=\sigma_{3}\left(-x_{1}-x_{2}-x_{3}-\varepsilon x_{4}-\alpha x_{5}\right), \\
& \dot{x}_{5}=\sigma_{4}\left(-x_{1}-x_{2}-x_{3}-\varepsilon x_{4}-\mu \alpha x_{5}\right),
\end{aligned}
$$

where the dots denote differentiation with respect to $\tau$ and $\phi(y)=\exp (-y)-1$.

Note that our model is nonsymmetric due to the presence of the exponential nonlinearity in (5a)-(5e). Therefore, the system cannot support symmetric orbits. In this mathematical modeling, we consider an exponential model instead of a piecewise linear model (PWL) of the oscillator as previously adopted by some authors [11-14]. In fact, the PWL model may experience different types of bifurcations compared to the exponential model which it approximates (first-order approximation) [8]. Furthermore, the exponential model is more tractable both numerically and analytically compared to PWL model. Then exponential model may be exploited to derive the exact bifurcation structure occurring in the controlled Colpitts oscillators. Extended discussions can be found in [15].

\section{Dynamical Properties}

3.1. Dissipative and Existence of Attractors. The state space of system (5a), (5b), (5c), (5d), and (5e) is five-dimensional. The vector field on the right-hand sides of system (6) is defined as

$$
v(x)=\left[\begin{array}{c}
v_{1}(x) \\
v_{2}(x) \\
v_{3}(x) \\
v_{4}(x) \\
v_{5}(x)
\end{array}\right]=\left[\begin{array}{c}
\sigma_{1}\left(x_{4}-\gamma \phi\left(x_{2}+x_{3}\right)\right) \\
x_{4} \\
\sigma_{2}\left(x_{4}-\gamma \phi\left(x_{2}\right)\right) \\
\sigma_{3}\left(-x_{1}-x_{2}-x_{3}-\varepsilon x_{4}-\alpha x_{5}\right) \\
\sigma_{4}\left(-x_{1}-x_{2}-x_{3}-\varepsilon x_{4}-\mu \alpha x_{5}\right)
\end{array}\right] .
$$

The divergence of the vector field $v$ is evaluated as

$$
\nabla v(x)=\frac{\partial v_{1}}{\partial x_{1}}+\frac{\partial v_{2}}{\partial x_{2}}+\frac{\partial v_{3}}{\partial x_{3}}+\frac{\partial v_{4}}{\partial x_{4}}+\frac{\partial v_{5}}{\partial x_{5}}=-\left(\sigma_{3} \varepsilon+\sigma_{4} \mu \alpha\right)
$$

In view of (7), it can easily be shown that system (5a), (5b), (5c), (5d), and (5e) is dissipative with an exponential rate $d v / d t=\exp \left(-\sigma_{3} \varepsilon-\sigma_{4} \mu \alpha\right)$. Then, in the dynamical system (5a), (5b), (5c), (5d), and (5e), a volume element $V_{0}$ is apparently contracted by the flow into a volume element $V_{0} \exp \left(-\sigma_{3} \varepsilon-\sigma_{4} \mu \alpha\right) t$ in time $t$. It means that each volume containing the trajectories of this dynamical system shrinks to zero as $t \rightarrow \infty$ as an exponential rate. Therefore, all these dynamical system orbits are eventually confined to a specific subset that has zero volume; namely, the asymptotic motion settles onto an attractor of this system [16].

3.2. Chaotic Behavior. Generally, two indicators are used to identify the type of transition leading to chaos. The first indicator is the bifurcation diagram which summarizes the different dynamical behaviours exhibited by the oscillator and the second is the largest one-dimensional (1D) numerical Lyapunov exponent defined by

$$
\lambda_{\max }=\lim _{t \rightarrow \infty}\left[\left(\frac{1}{t}\right) \ln (d(t))\right],
$$

where

$$
d(t)=\sqrt{\delta_{1}^{2}+\delta_{2}^{2}+\delta_{3}^{2}+\delta_{4}^{2}+\delta_{5}^{2}},
$$

and computed from the following variational equations obtained by perturbing the solutions of (5a), (5b), (5c), (5d), and (5e) as follows: $x_{1} \rightarrow x_{1}+\delta_{1}, x_{2} \rightarrow x_{2}+\delta_{2}$, $x_{3} \rightarrow x_{3}+\delta_{3}, x_{4} \rightarrow x_{4}+\delta_{4}$, and $x_{5} \rightarrow x_{5}+\delta_{5}$. $d(t)$ is the distance between neighbouring trajectories in the phase space. Asymptotically $d(t)=\exp \left(\lambda_{\max } t\right)$. Thus, if $\lambda_{\text {max }}>0$, neighbouring trajectories diverge and the state of the oscillator is chaotic. For $\lambda_{\max }<0$, these trajectories converge and the state of the oscillator is nonchaotic. The case $\lambda_{\text {max }}=0$ corresponds to the torus state of the oscillator.

In order to analyse the influence of the control parameter on the dynamics of the controlled two-stage Colpitts oscillator, we set $\sigma_{1}=1.250, \sigma_{2}=1.000, \sigma_{3}=2.770, \sigma_{4}=1.853$, $\varepsilon=0.875, \mu=1.562$, and $\gamma=2.692$ and only vary $\alpha$ in the domain $1 \leq \alpha \leq 2$. The system (5a), (5b), (5c), (5d), and (5e) is solved numerically to define routes to chaos in our model using the fourth-order Runge-Kutta algorithm. The initial values of the system (5a), (5b), (5c), (5d), and (5e) are arbitrarily taken as $\left[\begin{array}{lllll}0.001 & 0.001 & 0.001 & 0.001 & 0.001\end{array}\right]$. Bifurcation diagram and corresponding graph of 1D largest Lyapunov exponent are obtained. Sample results are provided in Figure 2 where a bifurcation diagram associated with the corresponding graph of largest 1D largest numerical Lyapunov exponent summarizes various scenarios leading to chaos in the controlled two-stage Colpitts oscillator. This bifurcation diagram is obtained by plotting the coordinate $x_{5}$ in terms of the control parameter $\alpha$. The positive value of $\lambda_{\text {max }}$ is the signature of chaotic oscillations of the oscillator. Note that the various transitions/routes to chaos observed in the controlled two-stage Colpitts oscillator are commonly observed in various nonlinear systems $[15,17,18]$ including the universal Chua circuit, phase-locked loops, and the transformer-coupled oscillators, just to name a few. This serves to justify the richness of the bifurcations in the controlled two-stage Colpitts oscillator and also the striking phenomena exhibited by such oscillators.

In order to study the effects of the transistor gain $\beta$ on the dynamics of the oscillator, we reconsidered system (2a), (2b), (2c), (2d), and (2e) with $\alpha_{F_{i}} \neq 1(i=1,2)$. Then the normalized circuit equations can be rewritten as follows:

$$
\begin{aligned}
& \dot{x}_{1}=\sigma_{1}\left(x_{4}-\alpha_{F_{1}} \gamma \phi\left(x_{2}+x_{3}\right)\right), \\
& \dot{x}_{2}=x_{4}+\left(1-\alpha_{F_{1}}\right) \gamma \phi\left(x_{2}+x_{3}\right)+\left(1-\alpha_{F_{2}}\right) \gamma \phi\left(x_{2}\right), \\
& \dot{x}_{3}=\sigma_{2}\left(x_{4}+\left(1-\alpha_{F_{1}}\right) \gamma \phi\left(x_{2}+x_{3}\right)-\alpha_{F_{2}} \gamma \phi\left(x_{2}\right)\right), \\
& \dot{x}_{4}=\sigma_{3}\left(-x_{1}-x_{2}-x_{3}-\varepsilon x_{4}-\alpha x_{5}\right), \\
& \dot{x}_{5}=\sigma_{4}\left(-x_{1}-x_{2}-x_{3}-\varepsilon x_{4}-\mu \alpha x_{5}\right),
\end{aligned}
$$




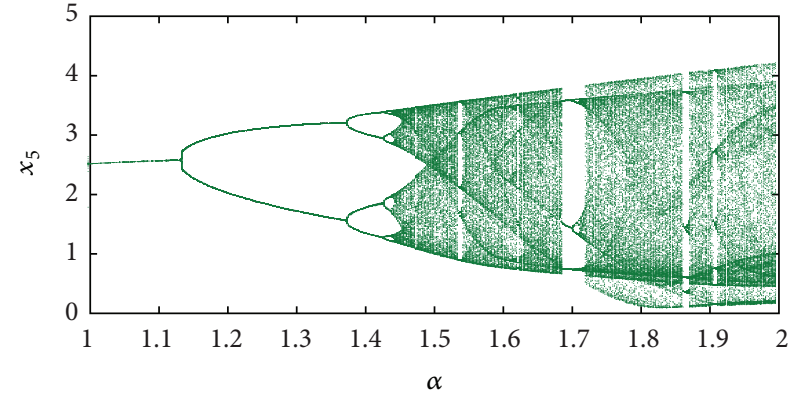

(a)

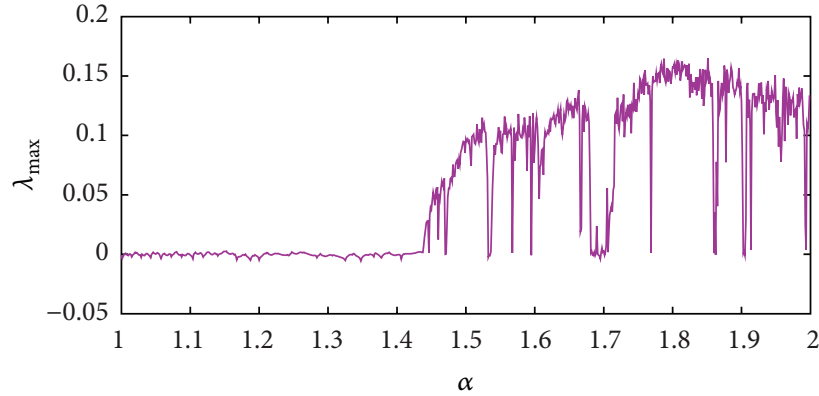

(b)

FIGURE 2: Bifurcation diagram of the controlled two-stage Colpitts oscillator (a) showing the state variable $x_{5}$ versus control parameter $\alpha$ and corresponding graph of $1 \mathrm{D}$ largest Lyapunov exponent (b). The parameters are $\sigma_{1}=1.250, \sigma_{2}=1.000, \sigma_{3}=2.770, \sigma_{4}=1.853, \varepsilon=0.875$, $\mu=1.562, \gamma=2.692$, and $\alpha_{F}=1$.

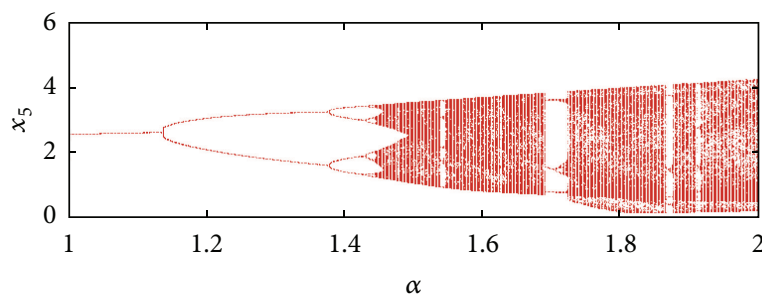

(a)

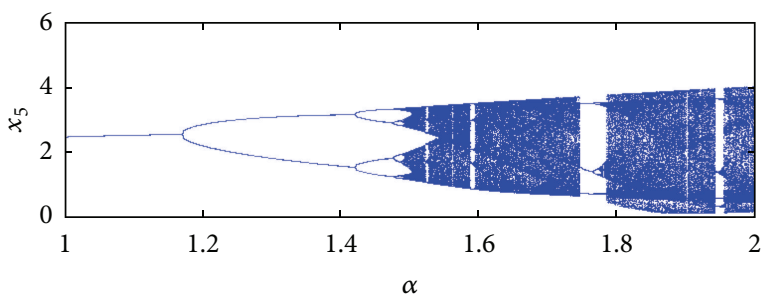

(b)

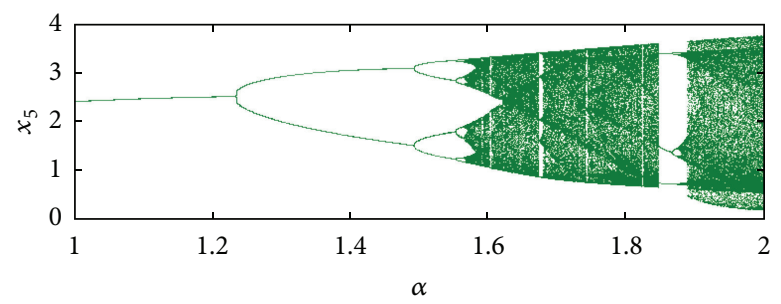

(c)

FIGURE 3: Bifurcation diagrams of the controlled chaotic Colpitts oscillator showing the effects of the transistor gain $\left(\beta=\alpha_{F} / 1-\alpha_{F}\right)$ on the dynamics of the oscillator plotted for (a) $\alpha_{F}=1$, (b) $\alpha_{F}=0.9803$, and (c) $\alpha_{F}=0.9876$. The rest of the system parameters are those in Figure 2 .

where the state variables and corresponding normalization are as defined previously. The relationship between the transistor gain $(\beta)$ and common base forward short-circuit gain of the transistor $\left(\alpha_{F}\right)$ is defined as

$$
\beta=\frac{\alpha_{F}}{1-\alpha_{F}} .
$$

We assume that the two transistors are equivalent; that is, $\left(\alpha_{F_{1}}=\alpha_{F_{2}}\right)$. According to (11), the numerical value of $\alpha_{F}$ is 0.9803 for $\beta=50$ and 0.987 for $\beta=80$ are used in the simulations. The effect of the transistor gain on the dynamics of the oscillator is illustrated by the bifurcation diagram of Figure 3. We observe that this diagram is similar to the one previously obtained with the ideal transistor model for $\alpha_{F_{i}}=$ $1(i=1,2)$ (see Figure 3(a)). Nevertheless, it is obvious that the value of $\left(\alpha_{F_{i}} \neq 1(i=1,2)\right)$ induces a horizontal stretching of the control parameter $\alpha$ (see Figures 3(b) and 3(c)).
Using the same parameters settings of Figure 2 and circuit parameters given in Figure 1, various numerical phase portraits and their corresponding power spectra were obtained confirming transitions/routes to chaos depicted previously (see Figure 4). The broadband noise-like power spectrum is the signature of a chaotic behaviour of the oscillator.

A blow-up of the bifurcation diagram of Figure 2 in the region of period 3 showing saddle-node bifurcation (SN), period-doubling (PD), and interior crisis (IC) is depicted in Figure 5.

\section{Experimental Study}

In this section, theoretical results obtained in the previous part are verified by carrying out an experimental study. The complex behaviour of the oscillator is investigated by monitoring a single variable resistor $R_{L}$, while keeping the rest of electronic components values constant. This section is 

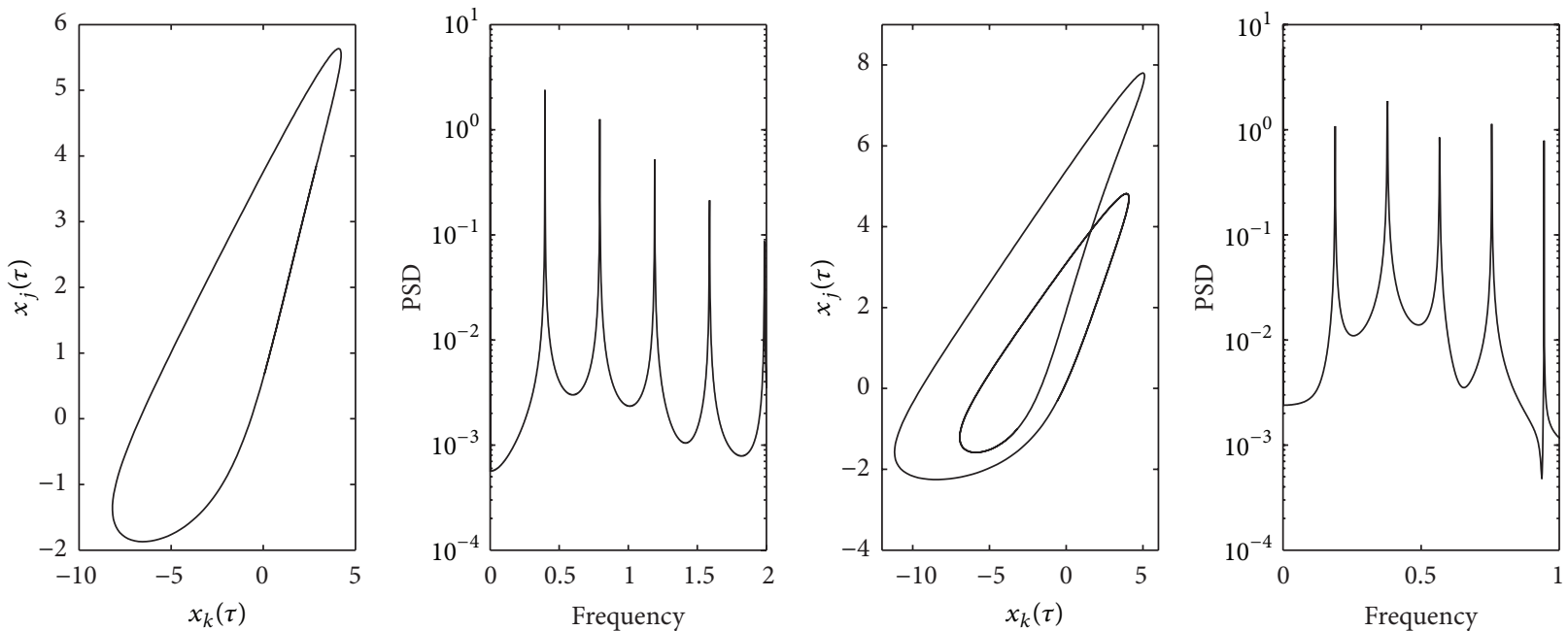

(a)

(b)
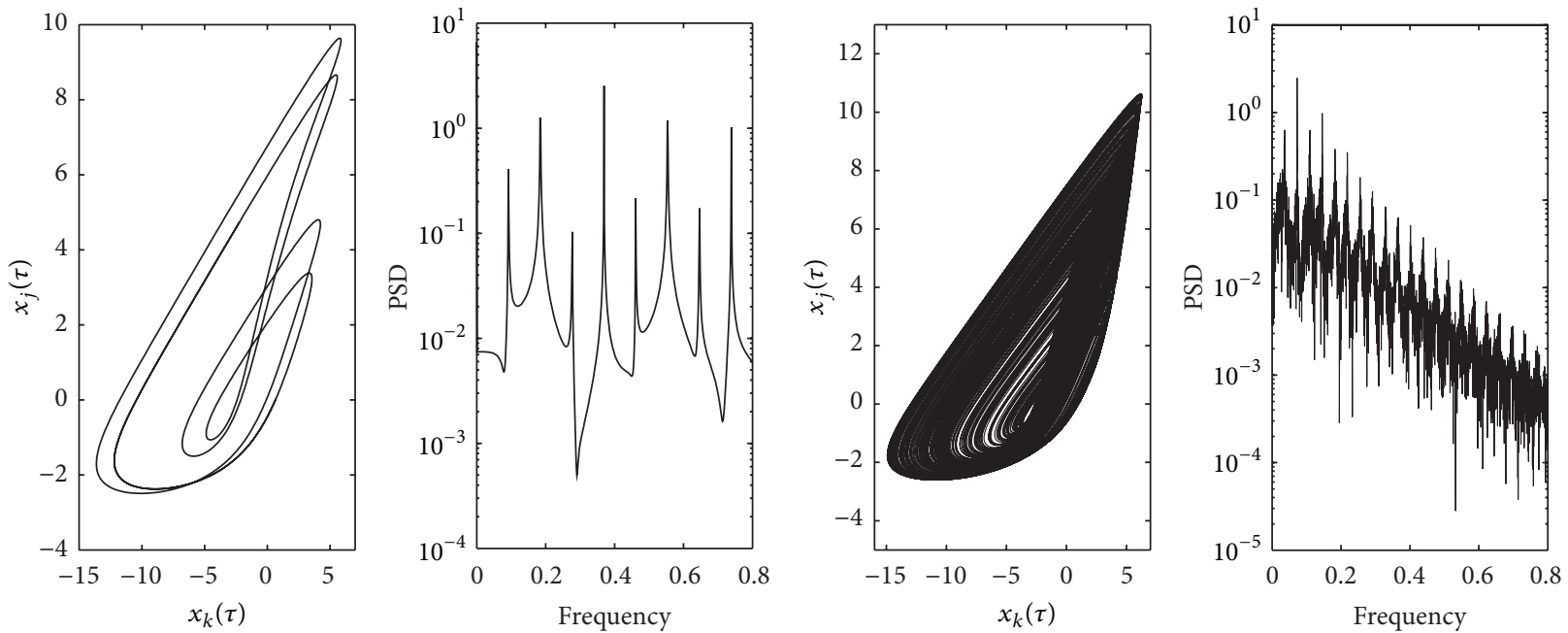

(c)

(d)
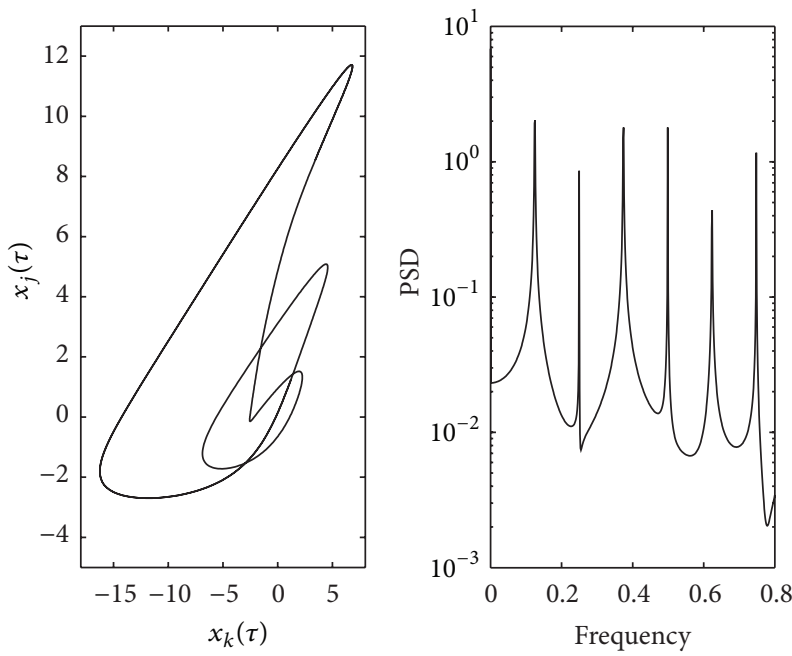

(e)
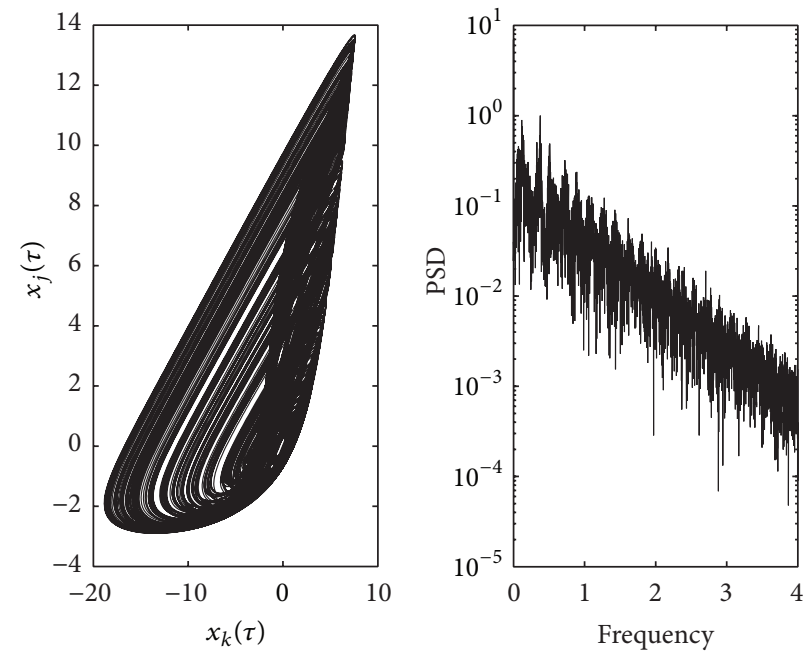

(f)

FIGURE 4: Computer generated phase portraits (left) of the system projected onto the plane $\left(x_{K}-x_{j}\right)$ showing routes to chaos in terms of control parameter $\alpha$ and corresponding power spectra (right): (a) period 1 for $\alpha=0.6$, (b) period 2 for $\alpha=1.3$, (c) period 4 for $\alpha=1.4$, (d) chaos for $\alpha=1.5$, (e) period 3 for $\alpha=1.68$, and (f) chaos for $\alpha=1.8$. The parameters are defined in text. Note that $x_{k}=x_{1}+x_{2}+x_{3}$ and $x_{j}=x_{2}+x_{3}$. 


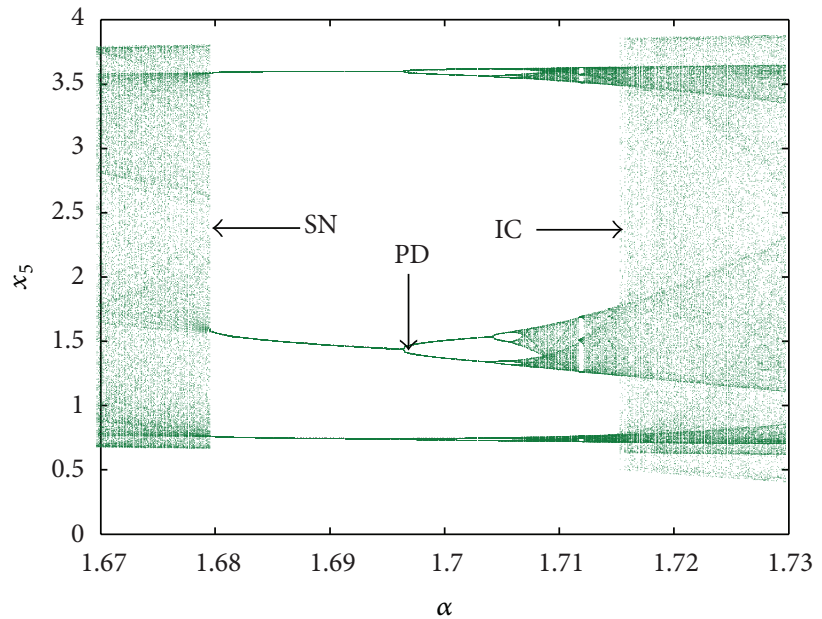

FIGURE 5: Blow-up of the bifurcation diagram of Figure 2 in the region of period 3 window showing saddle-node bifurcation (SN), perioddoubling (PD), and interior crisis (IC). The rest of the parameters are those in Figure 4.

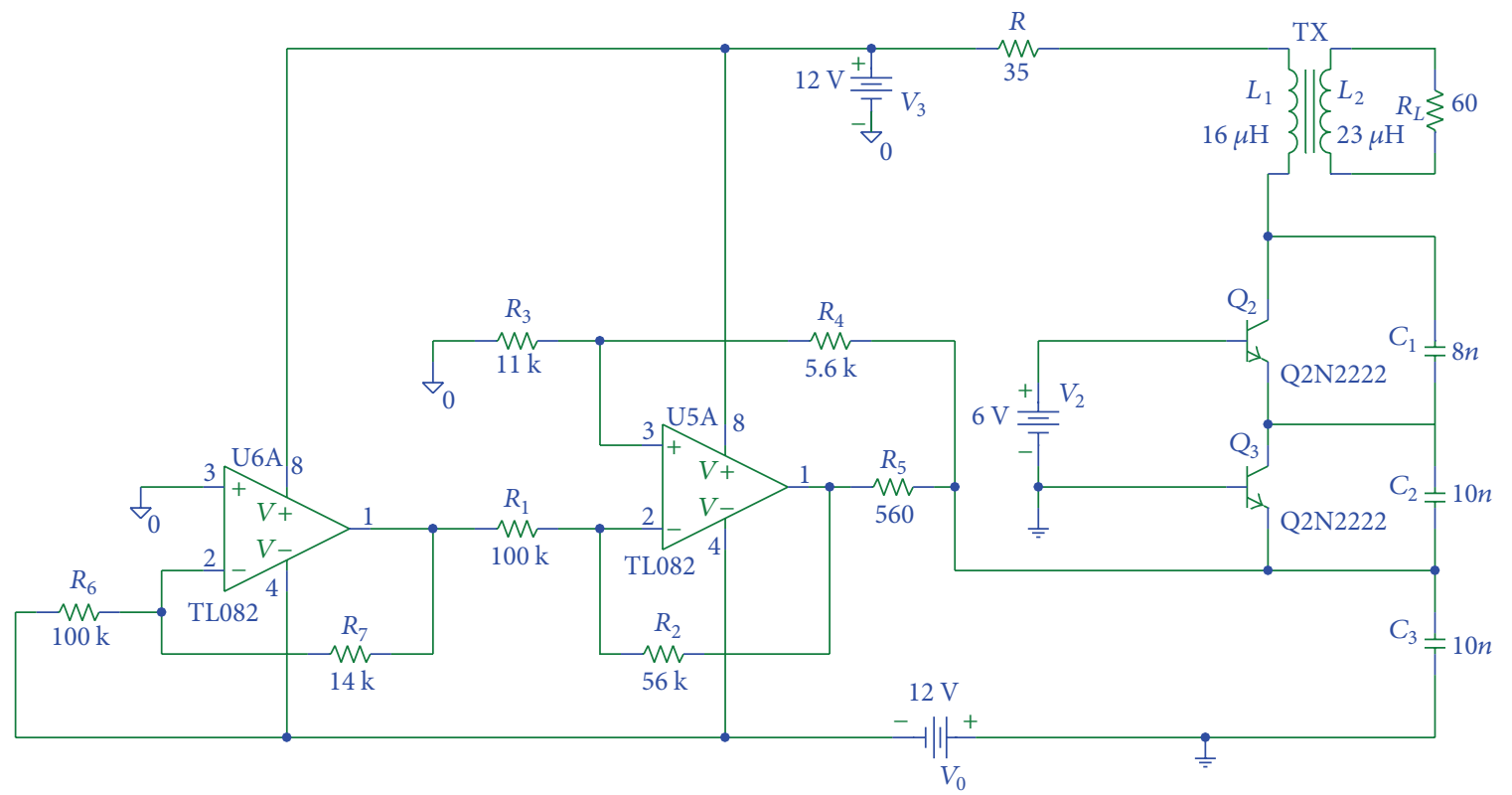

FIGURE 6: Experimental setup for measurements on the controlled two-stage Colpitts oscillator. The biasing current is constructed by the op. amplifiers (U1A and U2A) based network. Both $Q_{1}$ and $Q_{2}$ are 2 N2222 bipolar junction transistor type.

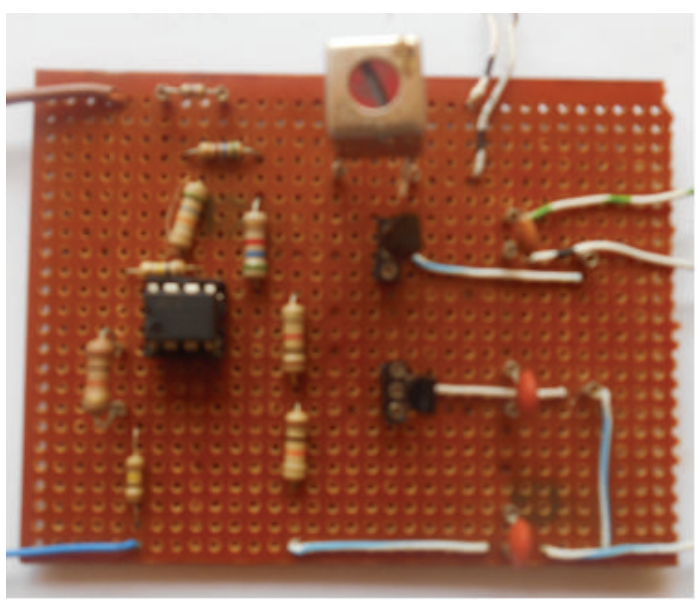

FIgURE 7: Photograph of the experimental controlled two-stage Colpitts oscillator circuit. 

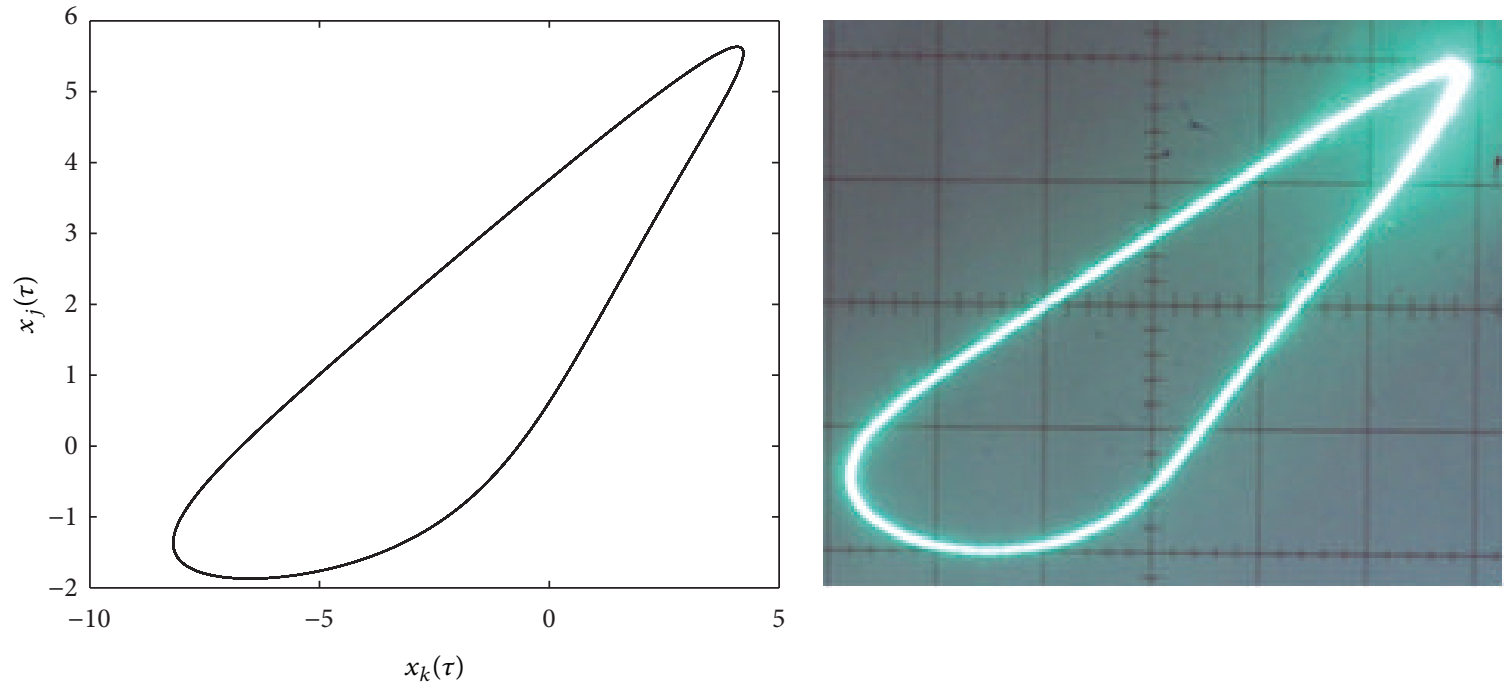

(a)
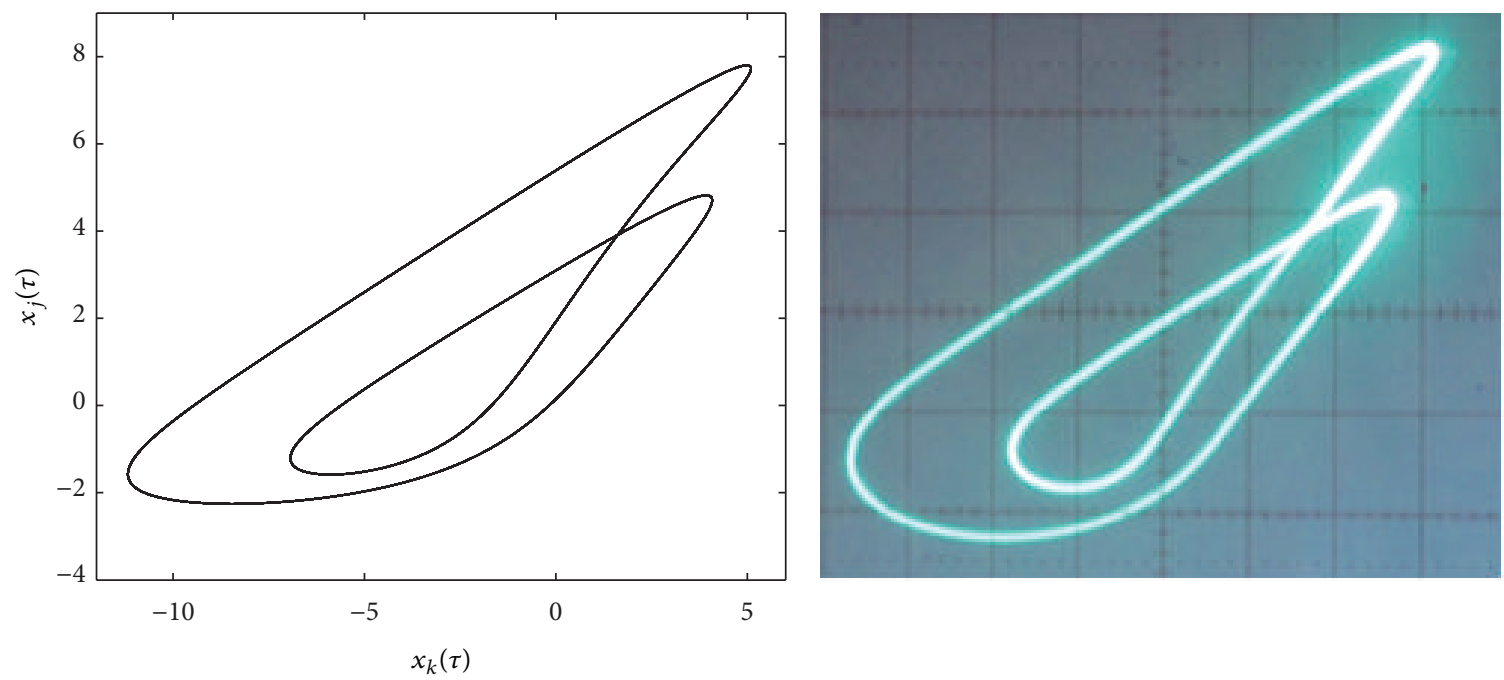

(b)
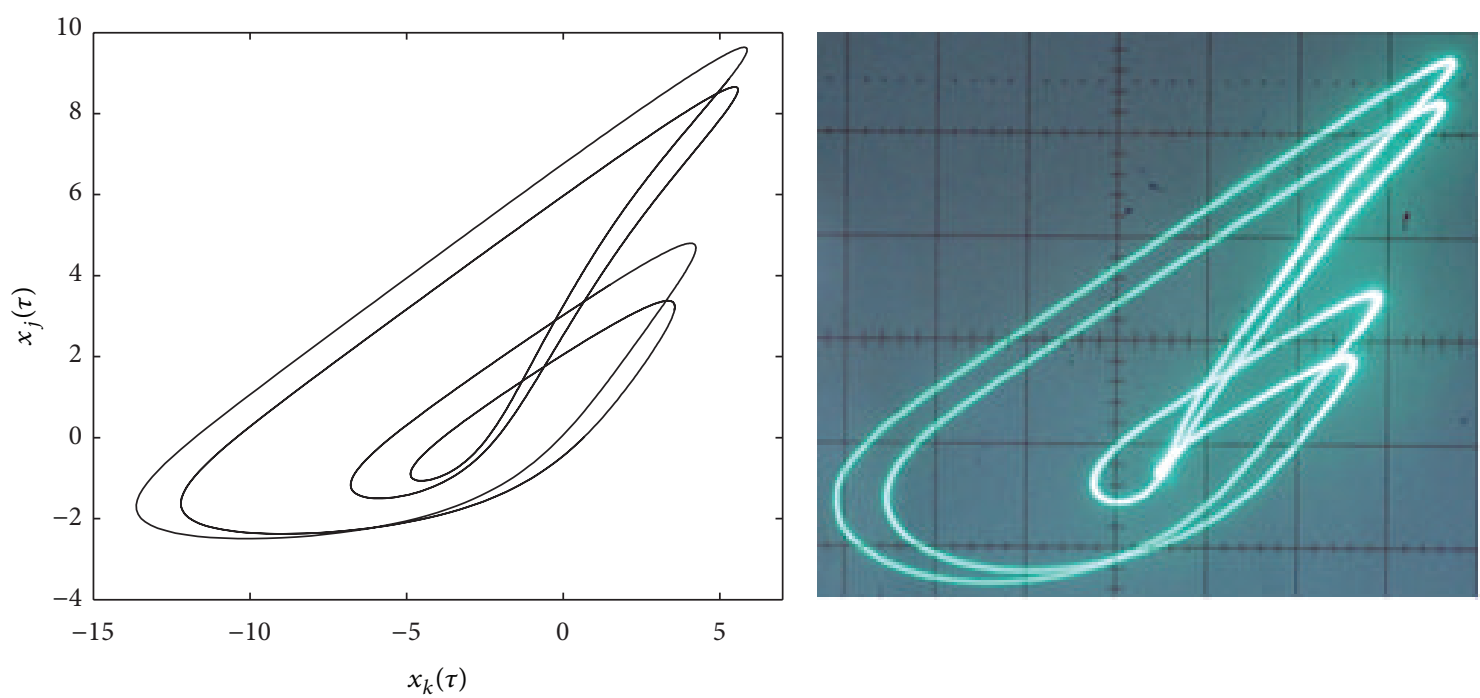

(c)

Figure 8: Continued. 

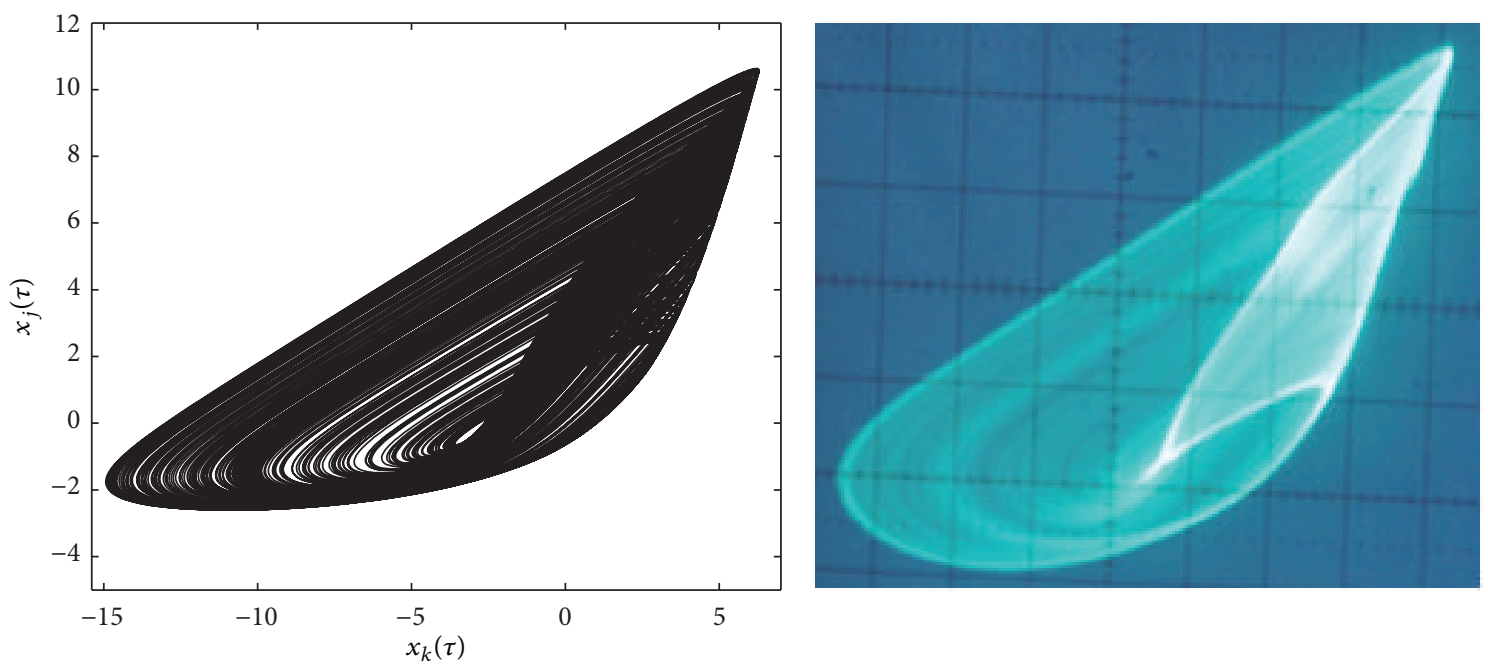

(d)
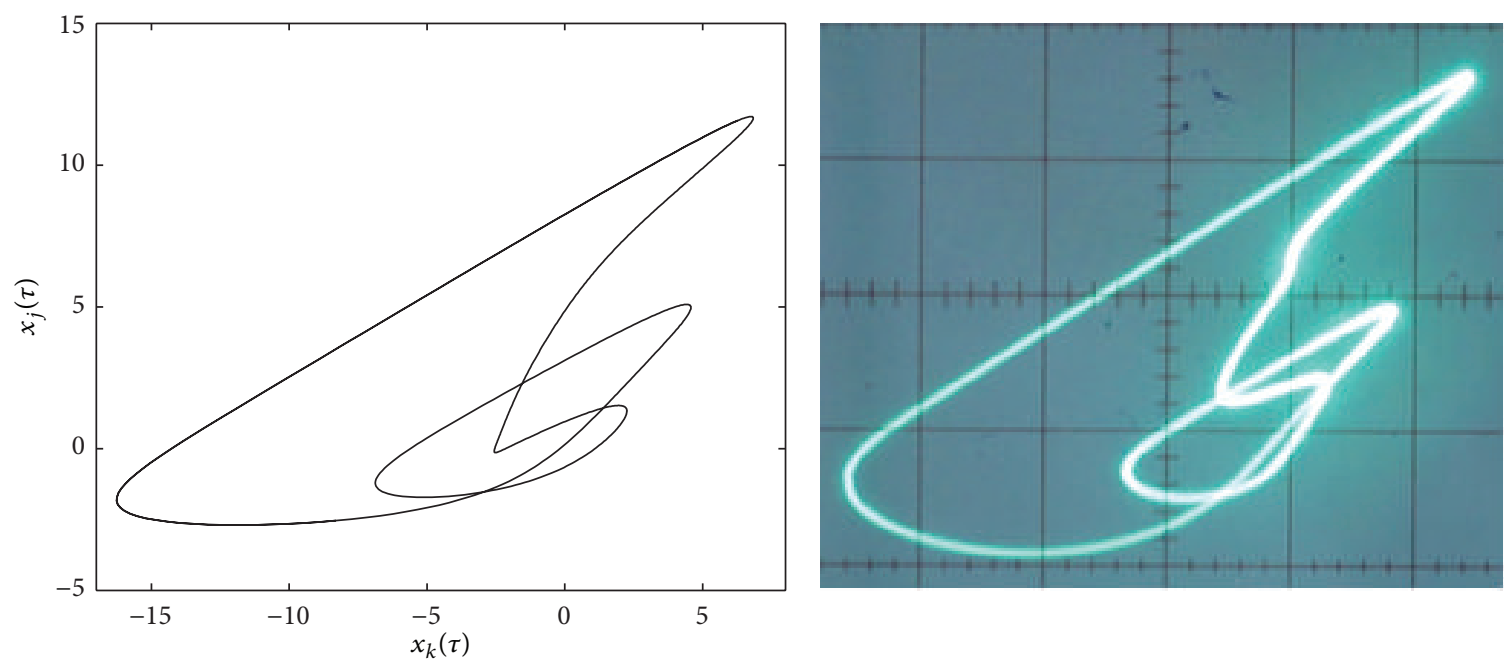

(e)
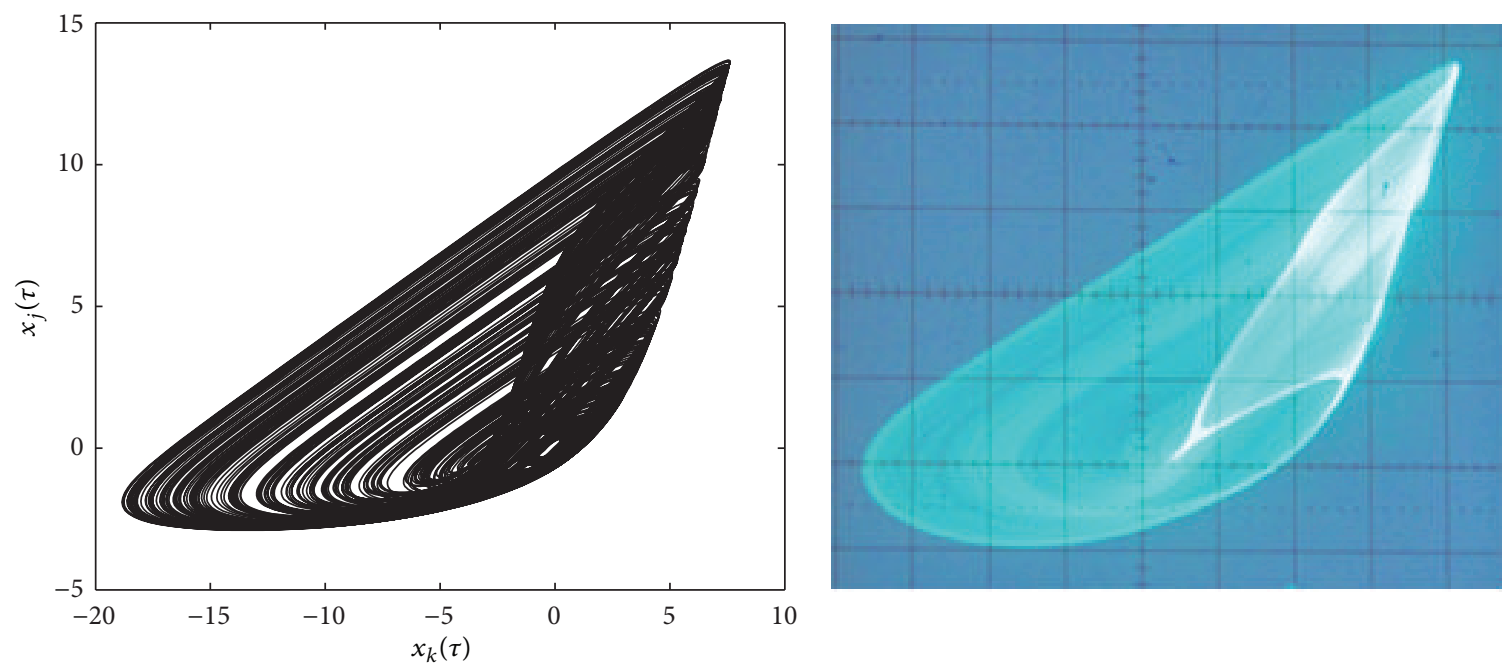

(f)

Figure 8: Experimental phase portraits (right) obtained from the circuit of Figure 8 using a dual trace oscilloscope in the $X Y$ mode. The collector voltage of BJT $Q_{1}\left(v_{c_{1}}+v_{c_{2}}+v_{c_{3}}\right)$ is connected to the $X$ input and the $Y$ channel displays the collector voltage of BJT $Q_{2}\left(v_{c_{2}}+v_{c_{3}}\right)$ : (a) period 1 for $R_{L}=60 \Omega$; (b) period 2 for $R_{L}=90 \Omega$; (c) period 4 for $R_{L}=102 \Omega$; (d) chaos for $R_{L}=120 \Omega$; (e) period 3 for $R_{L}=148 \Omega$; and (f) chaos for $R_{L}=160 \Omega$. Corresponding numerical phase portraits (right). 
also provided in order to evaluate the effects of the simplifying assumptions adopted during the modelling process on the real dynamics of the controlled two-stage Colpitts oscillator.

4.1. Design of the Experimental Setup. The experimental setup for measurements on the controlled two-stage Colpitts oscillator is depicted in Figure 6. This figure is carried out using operational amplifier (TL082), bipolar junction transistors (2N2222), variable transformer, high precision resistors, and capacitors with corresponding values fixed as above. The bias is provided by a $12 \mathrm{~V} \mathrm{DC}$ symmetric source. In order to insure good functioning of the circuit during experimental process, the ideal current source is replaced by the network consisting of the operational amplifier UA1 associated with five resistors $R_{i}(i=1,2, \ldots, 5)$. This configuration satisfies the following condition:

$$
\frac{R_{1}}{R_{2}}=\frac{R_{3}}{R_{4}+R_{5}} .
$$
[10]:

The expression of the ideal current source $I_{0}$ is as follows

$$
I_{0}=\frac{R_{2}}{R_{1} R_{5}} V_{i},
$$

where $V_{i}$ is the output voltage of the network (i.e., an inverting amplifier) using the operational amplifier UA2. According to (12) and (13), we derive the following relationship between the control voltage $V_{i}$ and the ideal current source $I_{0}$ as

$$
I_{0}=10^{-3} V_{i}
$$

The photograph of the experimental controlled two-stage Colpitts oscillator is presented in Figure 7.

4.2. Experimental Results. In this section, we analyse the effects of the variable resistor $\left(R_{L}\right)$ on the dynamics of the controlled two-stage Colpitts oscillator. When monitoring the single variable resistor $\left(R_{L}\right)$, it is found that the electronic circuit experiences a rich and striking dynamical behaviour and various types of bifurcation. Some sample phase portraits obtained experimentally are shown in Figure 8. This figure (right side) presents the real dynamics of controlled twostage Colpitts oscillator. It is observed in Figure 8 that the real circuit presents the same bifurcation scenarios as shown using analytical methods (period $1 \rightarrow$ period $2 \rightarrow$ period $4 \rightarrow$ chaos $\rightarrow$ period $3 \rightarrow$ chaos). A very good qualitative agreement is obtained between numerical (left side of Figure 8) and experimental results (right side of Figure 8).

\section{Conclusion}

This paper has introduced and investigated the dynamics of the new controlled two-stage Colpitts oscillator. The proposed oscillator in its regular/periodic state can be used for instrumentation in laboratory. In its irregular state, it can also be exploited as high frequency chaotic signals generators suitable for chaos based communication. The dynamics of the oscillator is easily controlled via a single variable resistor. The circuit diagram of the oscillator was presented and the modeling process using exponential nonlinearities was performed to derive the set of coupled first-order nonlinear ordinary differential equations describing the behavior of the oscillator. Various bifurcation diagrams associated with their graph of 1D largest numerical Lyapunov exponent were obtained showing transitions/routes to chaos in terms of the control parameter (variable resistor). Theoretical/numerical and experimental results were compared and a very good agreement was observed. It was found that the oscillator moves from the state of fixed point motion to chaos via the usual paths of period-doubling and interior crisis routes when monitoring the control parameter (variable resistor $R_{L}$ ) in tiny steps.

\section{Conflict of Interests}

The authors declare that there is no conflict of interests regarding the publication of this paper.

\section{References}

[1] T. Banerjee and B. C. Sarkar, "Chaos, intermittency and control of bifurcation in a ZC2-DPLL," International Journal of Electronics, vol. 96, no. 7, pp. 717-732, 2009.

[2] M. P. Kennedy, "Chaos in the colpitts oscillator," IEEE Transactions on Circuits and Systems I: Fundamental Theory and Applications, vol. 41, no. 11, pp. 771-774, 1994.

[3] C. Wegener and M. P. Kennedy, "RF chaotic Colpitts oscillator," in Proceedings of an International Workshop on Nonlinear Dynamics of Electronic Systems, pp. 255-258, Dublin, Ireland, 1995.

[4] G. Mykolaitis, A. Tamasevicius, and S. Bumeliene, "Two-stage chaotic Colpitts oscillator for the UHF range," Elektronika ir Elektrotechnika, vol. 53, no. 4, pp. 13-15, 2004.

[5] J. Kengne, J. C. Chedjou, V. A. Fono, and K. Kyamakya, "On the analysis of bipolar transistor based chaotic circuits: case of a two-stage colpitts oscillator," Nonlinear Dynamics, vol. 67, no. 2, pp. 1247-1260, 2012.

[6] A. Tamaševičius, G. Mykolaitis, S. Bumeliene, A. Čenys, A. N. Anagnostopoulos, and E. Lindberg, "Two-stage chaotic colpitts oscillator," Electronics Letters, vol. 37, no. 9, pp. 549-551, 2001.

[7] A. Tamaševičius, S. Bumeliene, and E. Lindberg, "Improved chaotic Colpitts oscillator for ultrahigh frequencies," Electronics Letters, vol. 40, no. 25, pp. 1569-1570, 2004.

[8] J. Kengne, J. C. Chedjou, G. Kenne, and K. Kyamakya, "Dynamical properties and chaos synchronization of improved Colpitts oscillators," Communications in Nonlinear Science and Numerical Simulation, vol. 17, no. 7, pp. 2914-2923, 2012.

[9] V. K. Tamba, H. B. Fotsin, J. Kengne, P. K. Talla, and T. F. Fonzin, "Complete switched adaptive modified function projective synchronization of two-stage chaotic Colpitts oscillators with uncertain parameters and external disturbances," Far East Journal of Dynamical Systems, vol. 21, no. 2, pp. 93-114, 2013.

[10] A. Buscarino, L. Fortuna, M. Frasca, and G. Sciuto, "Coupled inductors-based chaotic colpitts oscillator," International Journal of Bifurcation and Chaos, vol. 21, no. 2, pp. 569-574, 2011. 
[11] A. Čenys, A. Tamaševičius, A. Baziliauskas, R. Krivickas, and E. Lindberg, "Hyperchaos in coupled Colpitts oscillators," Chaos, Solitons and Fractals, vol. 17, no. 2-3, pp. 349-353, 2003.

[12] G.-H. Li, "Synchronization and anti-synchronization of Colpitts oscillators using active control," Chaos, Solitons and Fractals, vol. 26, no. 1, pp. 87-93, 2005.

[13] S. Bumeliene, A. Tamaševičius, G. Mykolaitis, A. Baziliauskas, and E. Lindberg, "Numerical investigation and experimental demonstration of chaos from two-stage Colpitts oscillator in the ultrahigh frequency range," Nonlinear Dynamics, vol. 44, no. 14, pp. 167-172, 2006.

[14] J. Y. Effa, B. Z. Essimbi, and J. M. Ngundam, "Synchronization of improved chaotic Colpitts oscillators using nonlinear feedback control," Nonlinear Dynamics, vol. 58, no. 1-2, pp. 39-47, 2009.

[15] G. M. Maggio, O. De Feo, and M. P. Kennedy, "Nonlinear analysis of the Colpitts oscillator and applications to design," IEEE Transactions on Circuits and Systems I: Fundamental Theory and Applications, vol. 46, no. 9, pp. 1118-1130, 1999.

[16] V. Sundarapandian and I. Pehlivan, "Analysis, control, synchronization, and circuit design of a novel chaotic system," Mathematical and Computer Modelling, vol. 55, no. 7-8, pp. 1904-1915, 2012.

[17] L. O. Chua, C. W. Wu, A. Huang, and G.-Q. Zhong, "Universal circuit for studying and generating chaos-part I: routes to chaos," IEEE Transactions on Circuits and Systems I: Fundamental Theory and Applications, vol. 40, no. 10, pp. 732-744, 1993.

[18] J. C. Chedjou, K. Kyamakya, I. Moussa, H.-P. Kuchenbecker, and W. Mathis, "Behavior of a self-sustained electromechanical transducer and routes to chaos," Journal of Vibration and Acoustics, Transactions of the ASME, vol. 128, no. 3, pp. 282-293, 2006. 


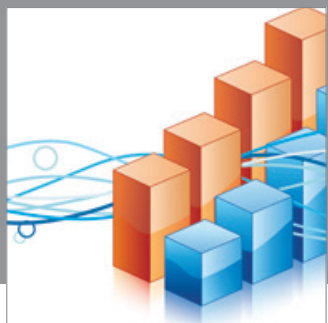

Advances in

Operations Research

mansans

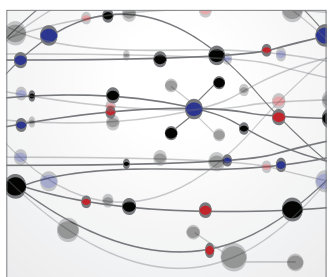

The Scientific World Journal
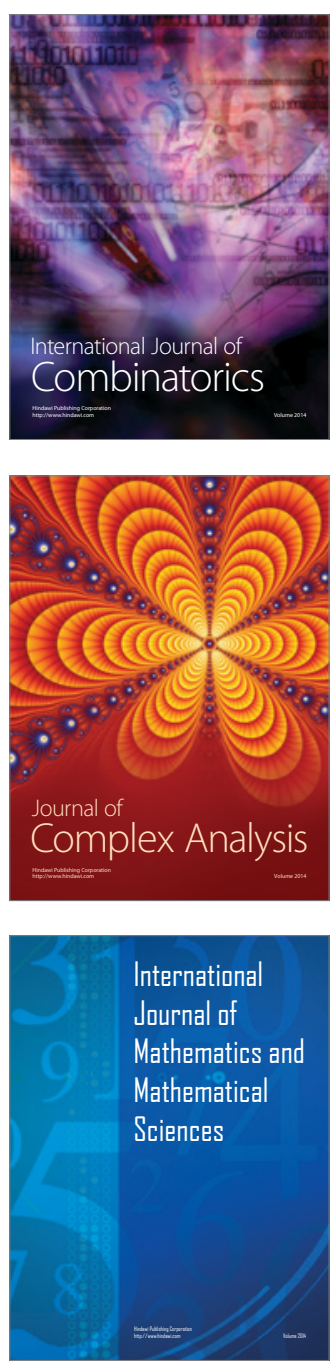
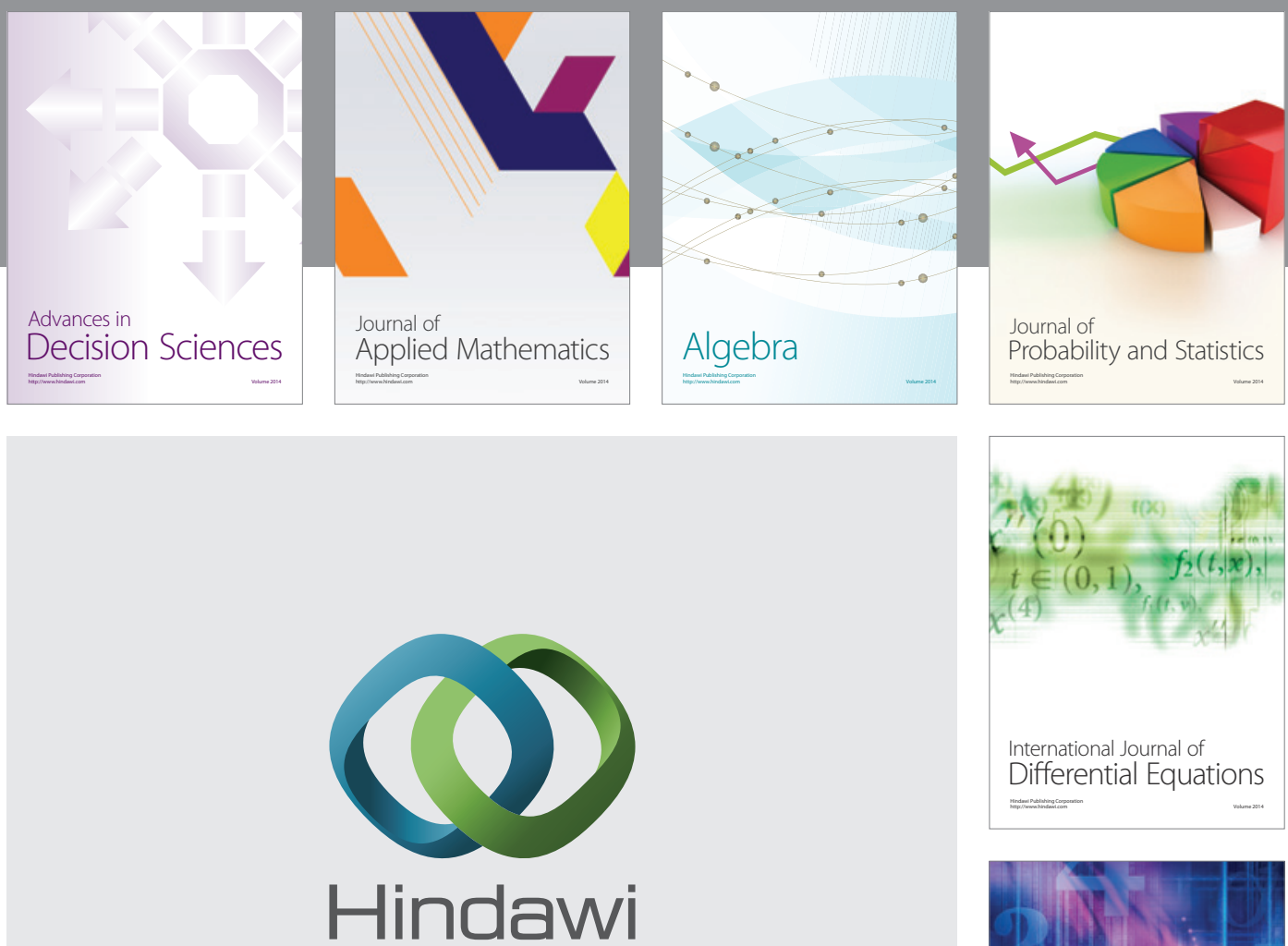

Submit your manuscripts at http://www.hindawi.com
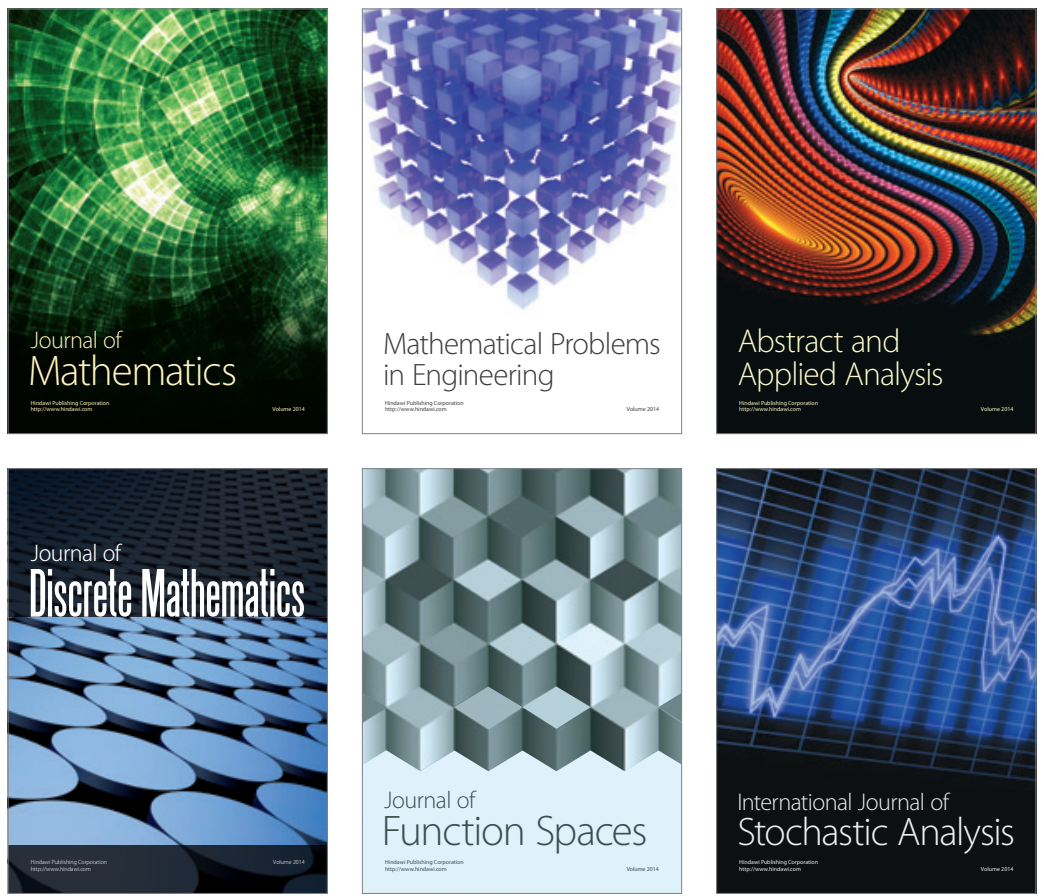

Journal of

Function Spaces

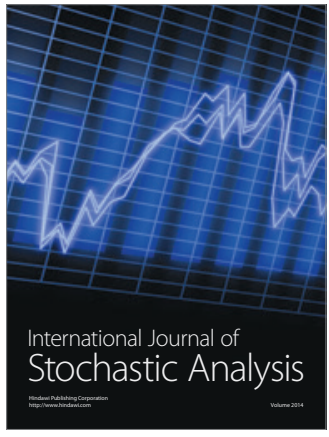

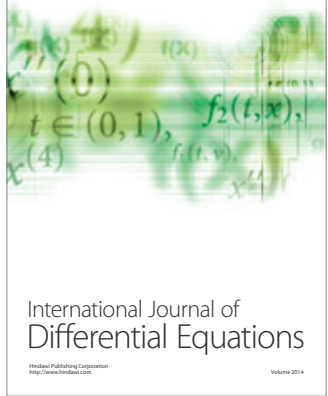
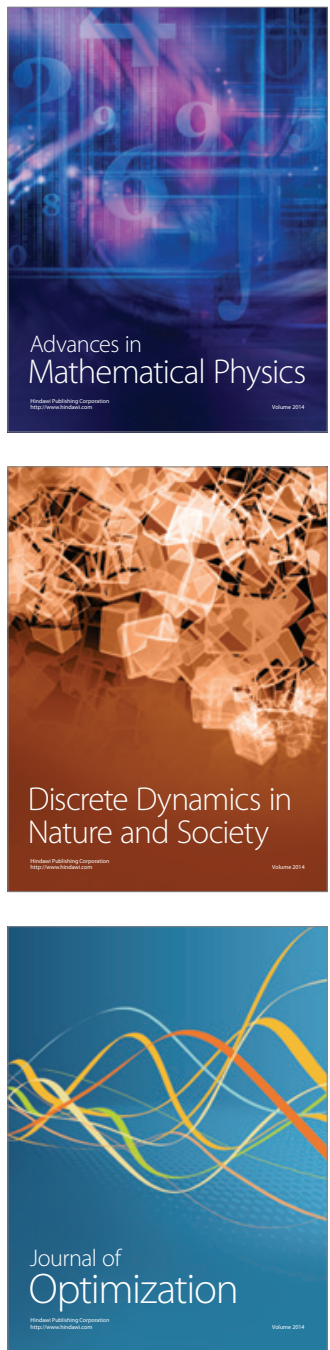